\title{
Endocytosis and Recycling of Tight Junction Proteins in Inflammation
}

\author{
Markus Utech, Rudolf Mennigen, and Matthias Bruewer \\ Department of General and Visceral Surgery, University Hospital Muenster, Waldeyerstraße 1, 48149 Muenster, Germany \\ Correspondence should be addressed to Matthias Bruewer, matthias.bruewer@ukmuenster.de
}

Received 19 July 2009; Accepted 28 October 2009

Academic Editor: Xue-Ru Wu

Copyright () 2010 Markus Utech et al. This is an open access article distributed under the Creative Commons Attribution License, which permits unrestricted use, distribution, and reproduction in any medium, provided the original work is properly cited.

A critical function of the epithelial lining is to form a barrier that separates luminal contents from the underlying interstitium. This barrier function is primarily regulated by the apical junctional complex (AJC) consisting of tight junctions (TJs) and adherens junctions (AJs) and is compromised under inflammatory conditions. In intestinal epithelial cells, proinflammatory cytokines, for example, interferon-gamma (IFN- $\gamma$ ), induce internalization of TJ proteins by endocytosis. Endocytosed TJ proteins are passed into early and recycling endosomes, suggesting the involvement of recycling of internalized TJ proteins. This review summarizes mechanisms by which TJ proteins under inflammatory conditions are internalized in intestinal epithelial cells and point out comparable mechanism in nonintestinal epithelial cells.

\section{Introduction}

The epithelial lining of different apparatuses functions as an important barrier that protects the underlying tissue compartments from antigens and bacterial products in the lumen. The barrier function in epithelial cells is regulated by the apical intercellular junctional complex referred to as the apical junctional complex (AJC). The major constituents of this complex are tight junctions (TJs) and subjacent adherens junctions (AJs). TJ represent a multiprotein complex consisting of transmembrane and cytosolic components $[1,2]$.

Various studies over the last decades have shown that endocytosis of AJC components occurs in response to physiological and pathological stimuli both in vitro and in vivo [3-14]. For example, proinflammatory cytokines, such as interferon-gamma (IFN- $\gamma$ ), induce endocytosis of TJ proteins in epithelial cells [15]. Moreover, prolonged cytokine simulation, as occurs in chronic inflammatory bowel disease, induces endocytosis of several TJ proteins [15-18]. In this review, we will highlight the possible biological roles and mechanisms of endocytosis of TJ proteins under inflammatory conditions in epithelial cells, and we will point out comparable mechanisms in nonepithelial cells.

\section{The Apical Junctional Complex: Tight and Adherens Junctions}

Intercellular adhesion in epithelial cells is mediated by cellcell junctions that include TJ, AJ, and desmosomes [1, 19]. The TJ is the most luminal component of the AJC, which also includes the $\mathrm{AJ}$. Both the $\mathrm{TJ}$ and $\mathrm{AJ}$ are represented by several transmembrane and cytosolic proteins $[1,2,19,20]$. The mechanical link between two plasma membranes of adjacent cells is given by the transmembrane proteins establishing an effective paracellular barrier to diffusion of fluids and solutes, whereas the cytosolic plaque proteins are responsible for organizing and positioning of the AJC [1]. In addition, the structural and functional integrity of the TJ depends on the presence of a perijunctional ring of actin and myosin which can also contribute to the regulation of paracellular permeability. Transmembrane proteins of the TJ include occludin, members of the claudin family, and junctional adhesion molecule- (JAM-) A. E-cadherin and members of the nectin family represent the major transmembrane components of epithelial AJ $[19,21]$. Cytosolic plaque proteins include members of the zonula occludens ( $\mathrm{ZO}$ ) protein family ( $\mathrm{ZO}-1, \mathrm{ZO}-2$, and $\mathrm{ZO}-3$ ) that bind to $\mathrm{TJ}$ transmembrane proteins and the underlying perijunctional F-actin ring $[2,22,23]$. 


\section{Endocytic Pathways}

Endocytosis is the process by which cells absorb molecules (such as proteins) from outside the cell by engulfing it with their cell membrane. This uptake mediates different elementary cellular mechanism such as signal transduction, cell metabolism, differentiation, defense, and motility [24]. It involves an extensive cellular machinery to mediate the formation of membrane transport vesicles to enable the internalization of plasma membrane structures and extracellular material [24]. Endocytosis pathways could be subdivided into three categories: namely, clathrin-mediated endocytosis, caveolar-mediated endocytosis, and macropinocytosis. Clathrin-mediated endocytosis is mediated by small vesicles that are mainly associated with the cytosolic protein clathrin. Clathrin-coated vesicles are found in virtually all cells and from domains of the plasma membrane termed clathrincoated pits. Caveolar-mediated endocytosis engrosses invagination of cholesterol-enriched microdomains within the plasma membrane that may contain a coat protein, caveolin. These structures are referred to as lipid rafts and/or caveolae. Macropinocytosis, which usually occurs from highly ruffled regions of the plasma membrane, is the invagination of the cell membrane to form a pocket, which then pinches off into the cell to form a vesicle filled with large volume of extracellular fluid and molecules within it. The vesicle then travels into the cytosol and fuses with other vesicles such as endosomes and lysosomes. Formation of all types of endocytic vesicles requires unique macromolecular machinery including enzymes, adaptors, and motor proteins [24, 25].

\section{Endocytosis of TJ in Intestinal Epithelial Cells}

Interestingly, the plasma membrane area around the AJC is both a frequent origin and destination of endocytotic vesicles [26-28]. Many proteins involved in the formation and sorting of vesicles are localized at the AJC, such as Rab3b [29], Rab8 [30], Rab13 [31], and its effector protein JRAB/MICAL-L2 [32], as well as a vesicle associated protein VAP-33 [33]. Not only AJC proteins but also various other plasma membrane proteins are delivered to the AJC area and then transported to their final destination at the apical plasma membrane [26-28].

Many independent studies demonstrated that proteins of the AJC are internalised by endocytotic processes under various conditions. Studies on embryological tissues revealed the endocytosis of several adherens and tight junction proteins during morphogenesis [34-36]. Beside embryological implications, various pathological states are associated with an increased endocytosis of AJC proteins. Massive internalisation of AJC proteins has been found during experimental pancreatitis, following irradiation of intestinal epithelium, and in patients with retinal dystrophy [37-39]. A wide range of pathogenic stimuli, including cytokines, bacteria, toxins, and oxidative stress, have been identified as strong inductors of TJ and AJ protein endocytosis. [3, 5-10, 13-15].

The mechanisms that regulate endocytosis of AJC protein internalization have been studied in detail over the last decade and it is not surprising, that all three classic pathways of endocytosis have been reported to be involved, depending on the stimulus used. This includes clathrinmediated endocytosis of both $\mathrm{TJ}$ and AJ proteins using a nonphysiological stimulus, calcium depletion, resulting in loss of cell polarity and cell detachment [40], macropinocytosis of transmembranous TJ but not AJ proteins using the proinflammatory cytokine IFN- $\gamma$ causing an increased paracellular permeability [41] and caveolar-mediated endocytosis of occludin but not other transmembranous TJ or AJ proteins by Escherichia coli toxin cytotoxic necrotizing factor1, which activates RhoA [10].

Ivanov summarized in detail three potential modes of endocytosis of AJC proteins with distinct consequences for intestinal barrier function [42]. The first is the physiological low-grade constitutive internalization of certain junctional proteins without affecting AJC integrity and function. This can be regarded as "house-keeping" involving the normal turnover of AJC proteins. Internalized junctional proteins may degraded via late endosomes (LEs), or may be delivered back to the plasma membrane via recycling endosomes (REs) $[4,11,12]$. The second is the selective internalization of $\mathrm{TJ}$ but not $\mathrm{AJ}$ proteins. Internalized $\mathrm{TJ}$ proteins can be recycled back to the plasma membrane via $\mathrm{RE}$. Due to the massive loss of TJ proteins at the AJC, this mechanism causes increased paracellular permeability, while the AJ-mediated cell-cell contacts remain intact. This mode has been observed under inflammatory conditions $[10,41,43,44]$. The third is the internalization of both TJ and AJ proteins. Again, internalized proteins can be degradated or recycled. As both TJ and AJ are affected, this mechanism leads to a loss of intestinal barrier function, and of both cell-cell contacts and cell polarity. This mode is observed in epithelial cells subjected to calcium depletion [40,45-47] or in cells treated with either platelet-derived growth factor [8] or oxygen radicals $[9,42]$.

Since endocytosis mediates disassembly of the epithelial AJC, it is important to understand which pathways remove junctional proteins from the cell surface under inflammatory conditions. Escherichia coli cytotoxic necrotizing factor-1 (CNF-1), induced the disassembling of occludin from the TJ via a Rho, Rac, and Cdc42 activation by a caveolarmediated endocytosis [10]. Immunogold electron as well as immunofluorescence/confocal microscopy experiments revealed occludin and caveolin-1 internalization in caveolarlike structures in CNF-treated intestinal epithelial cells [10]. While internalized occludin did not colocalize with LAMP1, some occludin colocalized with EEA1 and rab11. Internalized occludin also colocalized with EEA-1, a marker for early endosomes and rab 11, a marker for recycling endosomes. This suggests that a pool of internalized occludin might be recycled back to the membrane in Rab11-positive recycling endosomes without new synthesis after withdrawal of CNF1 [10]. CNF-1 also induces redistribution of claudin-1, JAM$\mathrm{A}$, and ZO-1; however, these TJ proteins were not colocalized with occludin and adherens junctions were not disrupted.

Using proinflammatory cytokines, for example, IFN- $\gamma$, that are increased in inflammatory bowel disease (IBD), our data strongly support the involvement of macropinocytosismediated pathway in endocytosis of the transmembranous 
proteins occludin, claudin-1, and JAM-A without affecting ZO-1 or AJ-proteins [15]. Pharmacological inhibitors that prevent formation of clathrin-coated pits or caveolinmediated endocytosis did not attenuate TJ endocytosis. However, two standard pharmacological approaches to examine the role of macropinocytosis-inhibition of $\mathrm{Na}^{+} / \mathrm{H}^{+}$exchanger (EIPA and DMA) $[48,49]$ and phosphatidylinositol-3-kinase (PI3K) using wortmannin- $[50,51]$ prevented internalisation of occludin, claudin-1, and JAM-A. Finally, internalized TJ proteins significantly colocalized with a marker of macropinocytosis, rhodamine-dextran. Collectively, these data strongly suggest that IFN- $\gamma$ induces internalization of epithelial TJ proteins by a macropinocytosislike process.

To determine the fate of IFN- $\gamma$-induced internalized TJ protein colocalization, experiments with $\mathrm{TJ}$ proteins and marker for specific compartments were performed in vitro. Immunofluorescence labeling and confocal microscopy clearly showed colocalization of internalized occludin, JAMA, and claudin-1 with markers of early/recycling endosomes, EEA-1, Rab4, and Rab11. Localization of occludin in vesicles containing these markers was confirmed on an ultra-structural level by immunogold labeling and electron microscopy. Internalization of TJ-proteins was not accompanied by their accelerated degradation. Interestingly, removal of IFN- $\gamma$ from culture medium resulted in the disappearance of intracellular pools of occludin, JAM-A, and claudin1 and reaccumulation of these proteins at the TJ [41]. Such recovery of TJ was sensitive to exocytosis inhibitor monensin but not to inhibition of de novo protein synthesis, suggesting the involvement of recycling of internalized TJ proteins.

Until recently, analysis of AJC endocytosis in response to defined stimuli had only been studied in vitro. However, recent work has shown that either T-cell activation or direct administration of recombinant tumor necrosis factor (TNF) or LIGHT, a TNF core family member (lymphotoxin-like inducible protein that competes with glycoprotein $\mathrm{D}$ for herpes virus entry on $\mathrm{T}$ cells), induced occludin internalization and barrier dysfunction in mice, whereas endocytosis of ZO1 , claudins, and adherens junction proteins did not occur $[52,53]$. Internalized occludin also contained caveolin-1, but not clathrin heavy chain. More importantly, inhibition of caveolar endocytosis, but not clathrin-mediated endocytosis or macropinocytosis, prevented occludin internalization and barrier loss [54].

Endocytosis of TJ proteins have been demonstrated during infectious diseases as well. Wroblewski found that Helicobacter pylori infection of cultured monolayers or hypergastrinemic mice was associated with endocytosis of occludin and a consequent loss of epithelial barrier function [55]. In active IBD proinflammatory cytokines, such as IFN- $\gamma$ and TNF-alpha (TNF- $\alpha$ ) are increased leading to a further compromise in epithelial barrier function [56]. This is associated with an internalization of occludin and JAMA in subapical vesicle-like structures [41] in active ulcerative colitis as well as in acute dextran sodium sulphate- (DSS-) induced colitis [57]. Occludin endocytosis without claudin endocytosis is similar to that induced by latrunculin $\mathrm{A}$ in vitro, but contrasts sharply with the other in vitro studies which generally showed internalization of occludin along with claudin, JAM-A, or other TJ proteins. While it remains to be characterized, these disparate results may well reflect the difference between acute or transient stimuli and chronic stimuli. Thus, the prolonged cytokine simulation may induce internalization of claudin proteins as well as occludin. This, in fact, has recently been observed in epithelia from patients with Crohn's disease $[16,18,29]$.

\section{Endocytosis of TJ in Nonintestinal Epithelial Cells}

The mechanisms of TJ protein endocytosis by inflammatory mediators is not only observed in intestinal epithelial cells. In a vitro model of blood-testis barrier (BTB), Xia et al. focused on the fate of TJ proteins in primary cultured Sertoli cells after transforming growth factor (TGF)- $\beta 3$ and TNF- $\alpha$ treatment. In intact TJ TGF- $\beta 3$ and TNF$\alpha$ significantly accelerated internalization of JAM-A, Ncadherin, and occludin. Using phenylarsine oxide (PAO) that blocks clathrin-mediated endocytosis TGF- $\beta 3$-induced protein internalization was inhibited [44]. As PAO is known as an unspecific pharmacological inhibition tool also blocking macropinocytosis, phagocytosis [58, 59], protein tyrosine phosphatase, and Rho GTPase activity $[60,61]$, clathrin was knocked down by siRNA to a level of $50-60 \%$. The knockdown of clathrin by siRNA significantly blocked the accelerated endocytosis of JAM-A induced by TGF- $\beta 3$ [44].

Furthermore in a vitro model of blood-brain barrier, the proinflammatory mediator CCL2 induced disassembly of TJ proteins occludin and claudin- 5 by caveolin-mediated endocytosis. This observation was also based on pharmacological inhibition of caveolin-mediated internalization of claudin-5 and occludin using a cholesterol-binding agent, filipin III, and a cholesterol solubilizing agent, methyl- $\beta$-cyclodextrin [43].

To determine the intracellular compartments of internalized TJ proteins localisation experiments with GFPlabeled claudin-5 and occludin and marker for early endosomes (EEA), recycling endosomes (Rab 4) and lysosomes (LAMP2) were performed. The results indicate that internalization of claudin-5 and occludin follows a recycling rather than a degradation pathway and that recycled proteins are a substrate for the reassembly of the TJ complex during bloodbrain barrier recovery [43].

\section{Conclusion}

Focusing on barrier function in inflammation, it is obvious that the disassembly of AJC is triggered by endocytosis of selective TJ proteins, thereby influencing AJC structure and permeability across the epithelium. Due to the selective internalized TJ proteins without influencing AJ proteins, the paracellular permeability can increase without disruption of the cell-cell contacts. In intestinal as well as in nonintestinal epithelial cell tight junctions are internalized via 
endozytosis and placed to early and recycling endosomes without degradation of these proteins. After withdrawal of inflammatory agents, these pooled TJ proteins can be recycled back to the TJ without synthesis of de novo proteins, thereby regenerating the barrier function. However, this possibly describes a general mechanism of regulating the paracellular permeability by internalization of TJ proteins under inflammatory conditions. A better understanding of the mechanisms of junctional internalization and recycling will likely provide new insights into the mechanisms of clinically altered barrier function in inflammatory conditions such as inflammatory bowel disease.

\section{References}

[1] S. Tsukita, M. Furuse, and M. Itoh, "Multifunctional strands in tight junctions," Nature Reviews Molecular Cell Biology, vol. 2, no. 4, pp. 285-293, 2001.

[2] L. Gonzalez-Mariscal, A. Betanzos, P. Nava, et al., "Tight junction proteins," Program of Biophysics Molecular Biology, vol. 81, no. 1, pp. 1-44, 2003.

[3] C. G. Kevil, N. Ohno, D. C. Gute, et al., "Role of cadherin internalization in hydrogen peroxide-mediated endothelial permeability," Free Radicals on Biology and Medicine, vol. 24, no. 6, pp. 1015-1022, 1998.

[4] T. L. Le, A. S. Yap, and J. L. Stow, "Recycling of E-cadherin: a potential mechanism for regulating cadherin dynamics," Journal of Cell Biology, vol. 146, no. 1, pp. 219-232, 1999.

[5] M. Lecuit, R. Hurme, J. Pizarro-Cerda, et al., "A role for alpha-and beta-catenins in bacterial uptake," Proceedings of the National Academy of Science of the USA, vol. 97, no. 18, pp. 10008-10013, 2000.

[6] B. P. Mcnamara, A. Koutsouris, C. B. O'connell, et al., "Translocated EspF protein from enteropathogenic Escherichia coli disrupts host intestinal barrier function," Journal of Clinical Investigation, vol. 107, no. 5, pp. 621-629, 2001.

[7] A. Nusrat, C. Von Eichel-Streiber, J. R. Turner, et al., "Clostridium difficile toxins disrupt epithelial barrier function by altering membrane microdomain localization of tight junction proteins," Infection Immunity, vol. 69, no. 3, pp. 1329-1336, 2001.

[8] N. S. Harhaj, A. J. Barber, and D. A. Antonetti, "Plateletderived growth factor mediates tight junction redistribution and increases permeability in MDCK cells," Journal of Cellular Physiology, vol. 193, no. 3, pp. 349-364, 2002.

[9] R. K. Rao, S. Basuroy, V. U. Rao, et al., "Tyrosine phosphorylation and dissociation of occludin-ZO-1 and E-cadherin-betacatenin complexes from the cytoskeleton by oxidative stress," Biochemistry Journal, vol. 368, part 2, pp. 471-481, 2002.

[10] A. M. Hopkins, S. V. Walsh, P. Verkade, et al., "Constitutive activation of Rho proteins by CNF-1 influences tight junction structure and epithelial barrier function," Journal of Cell Science, vol. 116, part 4, pp. 725-742, 2003.

[11] G. Izumi, T. Sakisaka, T. Baba, et al., "Endocytosis of Ecadherin regulated by $\mathrm{Rac}$ and $\mathrm{Cdc} 42$ small $\mathrm{G}$ proteins through IQGAP1 and actin filaments," Journal of Cell Biology, vol. 166, no. 2, pp. 237-248, 2004.

[12] M. Matsuda, A. Kubo, M. Furuse, et al., "A peculiar internalization of claudins, tight junction-specific adhesion molecules, during the intercellular movement of epithelial cells," Journal of Cell Science, vol. 117, part 7, pp. 1247-1257, 2004.
[13] D. Talavera, A. M. Castillo, M. C. Dominguez, et al., "IL8 release, tight junction and cytoskeleton dynamic reorganization conducive to permeability increase are induced by dengue virus infection of microvascular endothelial monolayers," Journal of General Virology, vol. 85, part 7, pp. 1801-1813, 2004.

[14] C. N. Wrobel, J. Debnath, E. Lin, et al., "Autocrine CSF-1R activation promotes Src-dependent disruption of mammary epithelial architecture," Journal of Cell Biology, vol. 165, no. 2, pp. 263-273, 2004.

[15] M. Bruewer, A. Luegering, T. Kucharzik, et al., "Proinflammatory cytokines disrupt epithelial barrier function by apoptosisindependent mechanisms," Journal of Immunology, vol. 171, no. 11, pp. 6164-6172, 2003.

[16] S. Prasad, R. Mingrino, K. Kaukinen, et al., "Inflammatory processes have differential effects on claudins 2, 3 and 4 in colonic epithelial cells," Laboratory Investigation, vol. 85, no. 9, pp. 1139-1162, 2005.

[17] C. R. Weber and J. R. Turner, "Inflammatory bowel disease: is it really just another break in the wall?" Gut, vol. 56, no. 1, pp. 6-8, 2007.

[18] S. Zeissig, N. Burgel, D. Gunzel, et al., "Changes in expression and distribution of claudin 2, 5 and 8 lead to discontinuous tight junctions and barrier dysfunction in active Crohn's disease," Gut, vol. 56, no. 1, pp. 61-72, 2007.

[19] O. W. Blaschuk and T. M. Rowlands, "Plasma membrane components of adherens junctions (review)," Molecular Membrane Biology, vol. 19, no. 2, pp. 75-80, 2002.

[20] K. Matter and M. S. Balda, "Signalling to and from tight junctions," Nature Reviews Molecular Cell Biology, vol. 4, no. 3, pp. 225-236, 2003.

[21] A. S. Yap, W. M. Brieher, and B. M. Gumbiner, "Molecular and functional analysis of cadherin-based adherens junctions," Annual Review of Cell and Developmental Biology, vol. 13, pp. 119-146, 1997.

[22] A. S. Fanning, B. J. Jameson, L. A. Jesaitis, et al., "The tight junction protein ZO-1 establishes a link between the transmembrane protein occludin and the actin cytoskeleton," Journal of Biological Chemistry, vol. 273, no. 45, pp. 2974529753, 1998.

[23] E. S. Wittchen, J. Haskins, and B. R. Stevenson, "Protein interactions at the tight junction. Actin has multiple binding partners, and ZO-1 forms independent complexes with ZO-2 and ZO-3," Journal of Biological Chemistry, vol. 274, no. 49, pp. 35179-35185, 1999.

[24] S. Mukherjee, R. N. Ghosh, and F. R. Maxfield, "Endocytosis," Physiological Reviews, vol. 77, no. 3, pp. 759-803, 1997.

[25] J. S. Bonifacino and B. S. Glick, "The mechanisms of vesicle budding and fusion," Cell, vol. 116, no. 2, pp. 153-166, 2004.

[26] D. Louvard, "Apical membrane aminopeptidase appears at site of cell-cell contact in cultured kidney epithelial cells," Proceedings of the National Academy of Sciences of the United States of America, vol. 77, no. 7, pp. 4132-4136, 1980.

[27] G. Kreitzer, J. Schmoranzer, S. H. Low, et al., "Threedimensional analysis of post-Golgi carrier exocytosis in epithelial cells," Nature Cell Biology, vol. 5, no. 2, pp. 126-136, 2003.

[28] R. Polishchuk, A. Di Pentima, and J. Lippincott-Schwartz, "Delivery of raft-associated, GPI-anchored proteins to the apical surface of polarized MDCK cells by a transcytotic pathway," Nature Cell Biology, vol. 6, no. 4, pp. 297-307, 2004. 
[29] E. Weber, G. Berta, A. Tousson, et al., "Expression and polarized targeting of a rab3 isoform in epithelial cells," Journal of Cell Biology, vol. 125, no. 3, pp. 583-594, 1994.

[30] L. A. Huber, S. Pimplikar, R. G. Parton, et al., "Rab8, a small GTPase involved in vesicular traffic between the TGN and the basolateral plasma membrane," Journal of Cell Biology, vol. 123, no. 1, pp. 35-45, 1993.

[31] A. M. Marzesco, I. Dunia, R. Pandjaitan, et al., "The small GTPase Rab13 regulates assembly of functional tight junctions in epithelial cells," Molecular Biological Cell, vol. 13, no. 6, pp. 1819-1831, 2002.

[32] T. Terai, N. Nishimura, I. Kanda, et al., "JRAB/MICAL-L2 is a junctional Rab13-binding protein mediating the endocytic recycling of occludin," Molecular Biological Cell, vol. 17, no. 5, pp. 2465-2475, 2006.

[33] L. A. Lapierre, P. L. Tuma, J. Navarre, et al., "VAP-33 localizes to both an intracellular vesicle population and with occludin at the tight junction," Journal of Cell Science, vol. 112, part 21, pp. 3723-3732, 1999.

[34] S. Polak-Charcon, J. Shoham, and Y. Ben-Shaul, "Tight junctions in epithelial cells of human fetal hindgut, normal colon, and colon adenocarcinoma," Journal of the National Cancer Institute, vol. 65, no. 1, pp. 53-62, 1980.

[35] J. R. Miller and D. R. Mcclay, "Characterization of the role of cadherin in regulating cell adhesion during sea urchin development," Developmental Biology, vol. 192, no. 2, pp. 323339, 1997.

[36] H. Oda, S. Tsukita, and M. Takeichi, "Dynamic behavior of the cadherin-based cell-cell adhesion system during Drosophila gastrulation," Developmental Biology, vol. 203, no. 2, pp. 435450, 1998.

[37] M. Porvaznik, "Tight junction disruption and recovery after sublethal $\gamma$ irradiation," Radiation Research, vol. 78, no. 2, pp. 233-250, 1979.

[38] R. B. Caldwell, L. A. Wade, and B. J. Mclaughlin, "A quantitative study of intramembrane changes during cell junctional breakdown in the dystrophic rat retinal pigment epithelium," Experimental Cell Research, vol. 150, no. 1, pp. 104-117, 1984.

[39] M. M. Lerch, M. P. Lutz, H. Weidenbach, et al., "Dissociation and reassembly of adherens junctions during experimental acute pancreatitis," Gastroenterology, vol. 113, no. 4, pp. 13551366, 1997.

[40] A. I. Ivanov, A. Nusrat, and C. A. Parkos, "Endocytosis of epithelial apical junctional proteins by a clathrin-mediated pathway into a unique storage compartment," Molecular Biology of the Cell, vol. 15, no. 1, pp. 176-188, 2004.

[41] M. Bruewer, M. Utech, A. I. Ivanov, et al., "Interferon-gamma induces internalization of epithelial tight junction proteins via a macropinocytosis-like process," The FASEB Journal, vol. 19, no. 8, pp. 923-933, 2005.

[42] A. I. Ivanov, A. Nusrat, and C. A. Parkos, "The epithelium in inflammatory bowel disease: potential role of endocytosis of junctional proteins in barrier disruption," Novartis Foundation Symposium Journal, vol. 263, pp. 115-124, 2004.

[43] S. M. Stamatovic, R. F. Keep, M. M. Wang, et al., "Caveolaemediated internalization of occludin and claudin-5 during CCL2-induced tight junction remodeling in brain endothelial cells," Journal of Biological Chemistry, vol. 284, no. 28, pp. 19053-19066, 2009.

[44] W. Xia, E. W. Wong, D. D. Mruk, et al., “TGF-beta3 and TNFalpha perturb blood-testis barrier (BTB) dynamics by accelerating the clathrin-mediated endocytosis of integral membrane proteins: a new concept of BTB regulation during spermatogenesis," Development Biology, vol. 327, no. 1, pp. 4861, 2009.

[45] M. L. Troxell, Y. T. Chen, N. Cobb, et al., "Cadherin function in junctional complex rearrangement and posttranslational control of cadherin expression," American Journal of Pathology, vol. 276, no. 2, part 1, pp. C404-C418, 1999.

[46] B. Rothen-Rutishauser, F. K. Riesen, A. Braun, et al., "Dynamics of tight and adherens junctions under EGTA treatment," Journal of Membrane Biology, vol. 188, no. 2, pp. 151-162, 2002.

[47] A. I. Ivanov, I. C. Mccall, C. A. Parkos, et al., "Role for actin filament turnover and a myosin II motor in cytoskeletondriven disassembly of the epithelial apical junctional complex," Molecular Biological Cell, vol. 15, no. 6, pp. 2639-2651, 2004.

[48] M. A. West, M. S. Bretscher, and C. Watts, "Distinct endocytotic pathways in epidermal growth factor-stimulated human carcinoma A431 cells," Journal of Cell Biology, vol. 109, no. 6, part 1, pp. 2731-2739, 1989.

[49] O. Meier, K. Boucke, S. V. Hammer, et al., "Adenovirus triggers macropinocytosis and endosomal leakage together with its clathrin-mediated uptake," Journal of Cell Biology, vol. 158, no. 6, pp. 1119-1131, 2002.

[50] N. Araki, M. T. Johnson, and J. A. Swanson, "A role for phosphoinositide 3-kinase in the completion of macropinocytosis and phagocytosis by macrophages," Journal of Cell Biology, vol. 135, no. 5, pp. 1249-1260, 1996.

[51] M. Amyere, M. Mettlen, P. Van Der Smissen, et al., "Origin, originality, functions, subversions and molecular signalling of macropinocytosis," International Journal of Medical Microbiology, vol. 291, no. 6-7, pp. 487-494, 2002.

[52] D. R. Clayburgh, T. A. Barrett, Y. Tang, et al., "Epithelial myosin light chain kinase-dependent barrier dysfunction mediates T cell activation-induced diarrhea in vivo," Journal of Clinical Investigation, vol. 115, no. 10, pp. 2702-2715, 2005.

[53] D. R. Clayburgh, M. W. Musch, M. Leitges, et al., "Coordinated epithelial NHE3 inhibition and barrier dysfunction are required for TNF-mediated diarrhea in vivo," Journal of Clinical Investigation, vol. 116, no. 10, pp. 2682-2694, 2006.

[54] B. T. Schwarz, F. Wang, L. Shen, et al., "LIGHT signals directly to intestinal epithelia to cause barrier dysfunction via cytoskeletal and endocytic mechanisms," Gastroenterology, vol. 132, no. 7, pp. 2383-2394, 2007.

[55] L. E. Wroblewski, L. Shen, S. Ogden, et al., "Helicobacter pylori dysregulation of gastric epithelial tight junctions by urease-mediated myosin II activation," Gastroenterology, vol. 136, no. 1, pp. 236-246, 2009.

[56] C. Fiocchi, "Inflammatory bowel disease: etiology and pathogenesis," Gastroenterology, vol. 115, no. 1, pp. 182-205, 1998.

[57] R. Mennigen, K. Nolte, E. Rijcken, et al., "Probiotic mixture VSL\#3 protects the epithelial barrier by maintaining tight junction protein expression and preventing apoptosis in a murine model of colitis," American Journal of Physiology Gastrointestinal and Liver Physiology, vol. 296, no. 5, pp. G1140-G1149, 2009.

[58] S. C. Frost, M. D. Lane, and E. M. Gibbs, "Effect of phenylarsine oxide on fluid phase endocytosis: further evidence for activation of the glucose transporter," Journal of Cellular Physiology, vol. 141, no. 3, pp. 467-474, 1989. 
[59] P. Massol, P. Montcourrier, J. C. Guillemot, et al., "Fc receptormediated phagocytosis requires CDC42 and Rac1," EMBO Journal, vol. 17, no. 21, pp. 6219-6229, 1998.

[60] S. F. Retta, S. T. Barry, D. R. Critchley, et al., "Focal adhesion and stress fiber formation is regulated by tyrosine phosphatase activity," Cell Research, vol. 229, no. 2, pp. 307-317, 1996.

[61] R. Gerhard, H. John, K. Aktories, et al., "Thiol-modifying phenylarsine oxide inhibits guanine nucleotide binding of Rho but not of Rac GTPases," Molecular Pharmacology, vol. 63, no. 6, pp. 1349-1355, 2003. 

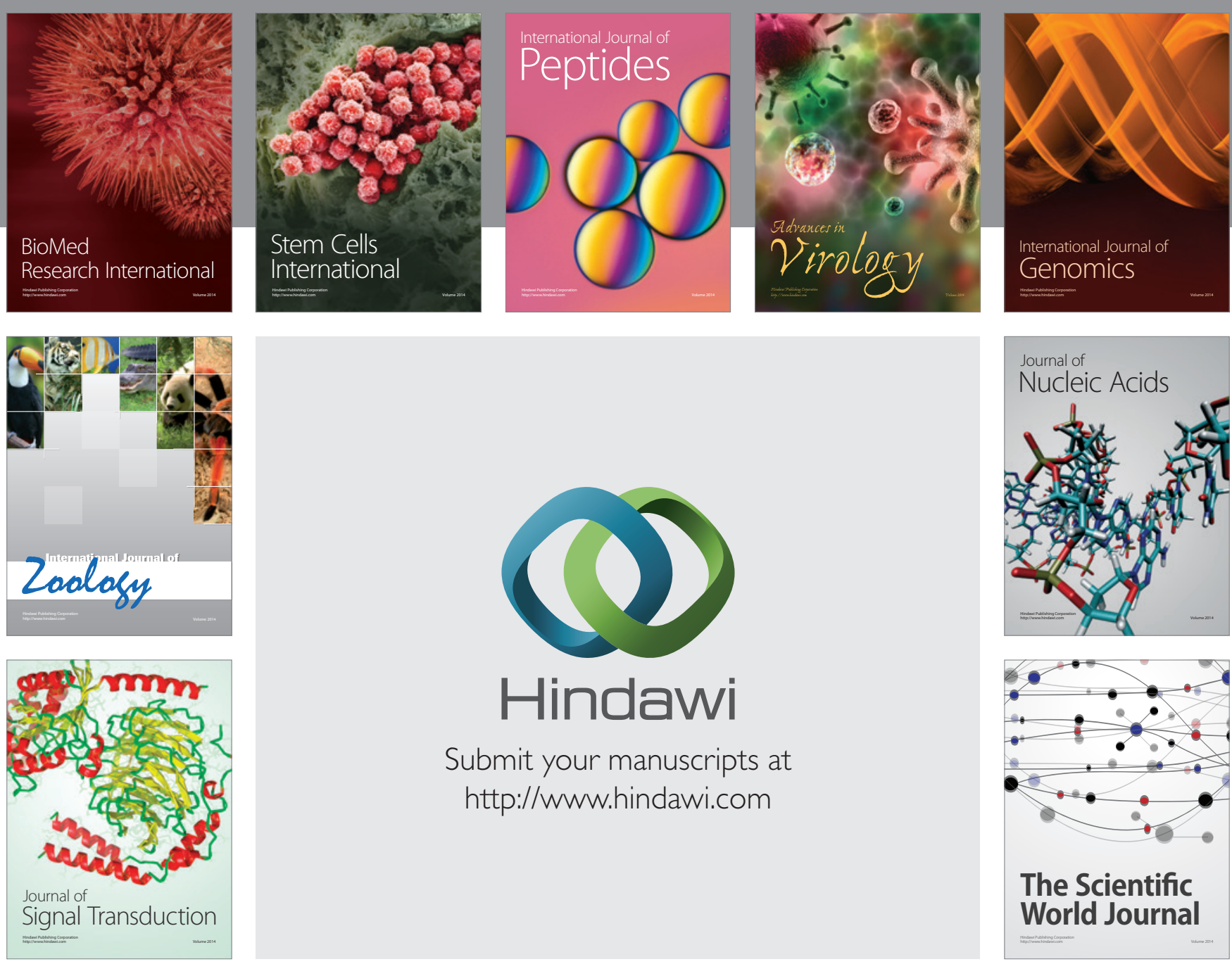

Submit your manuscripts at

http://www.hindawi.com
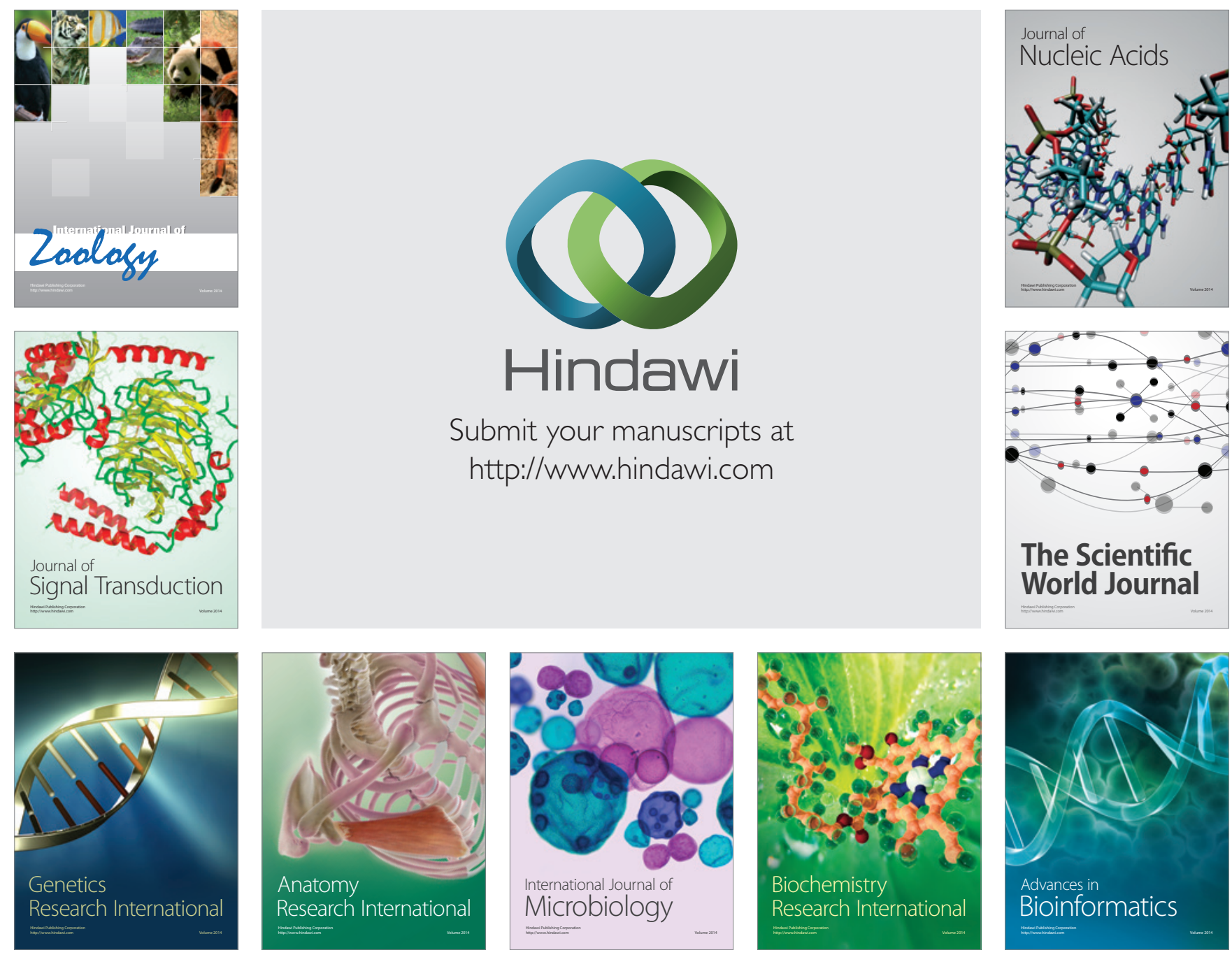

The Scientific World Journal
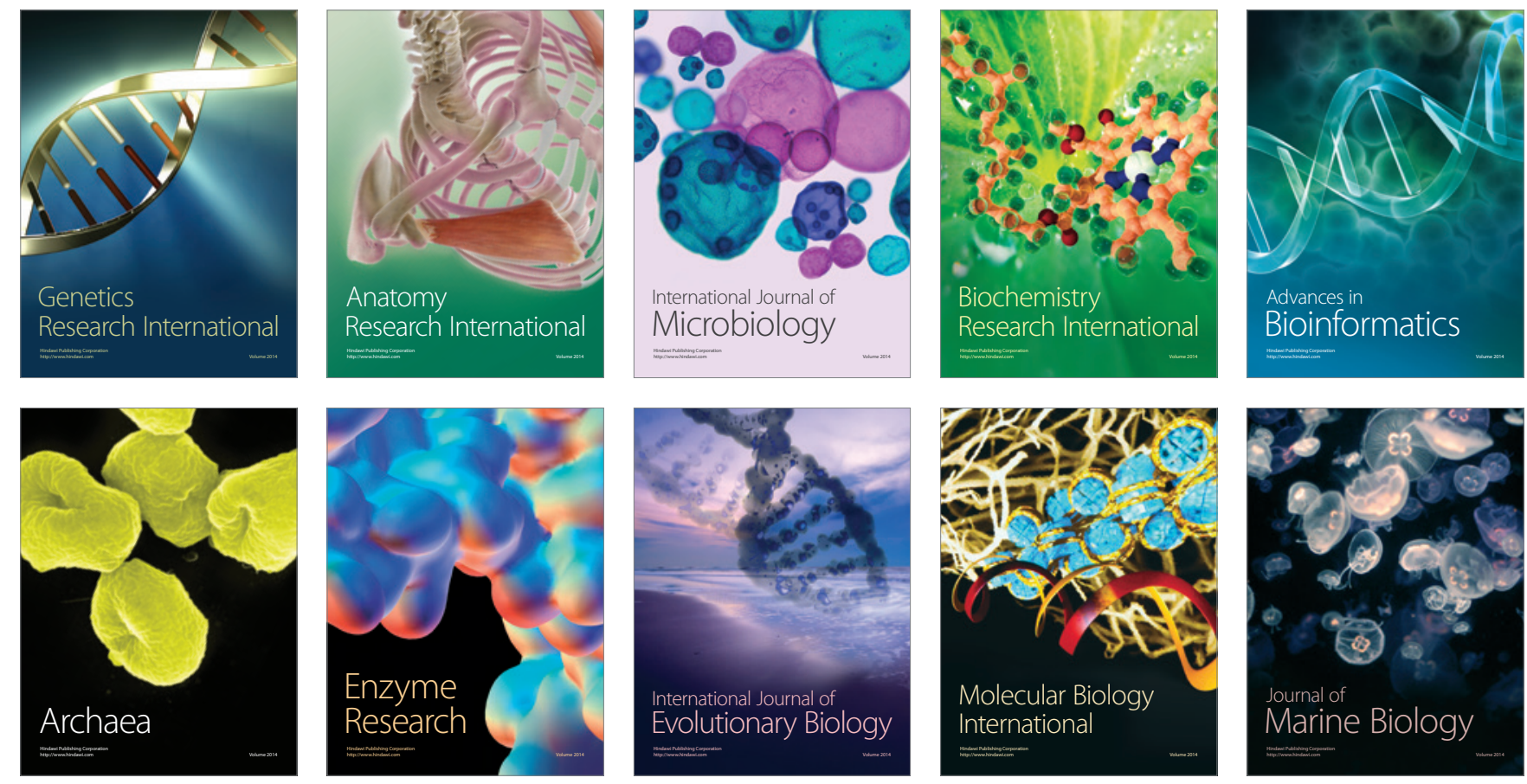